\title{
RSSA MDCT Essentials Course 2011
}

\section{Sandton Convention Centre, Johannesburg, 26 - 28 August 2011}

\author{
Oral presentations \\ (alphabetical order according to presenting author (bold font))
}

The role of multi-detector CT angiography as an adjunctive tool in the evaluation of paediatric cardiac disease in an African setting experiences at Red Cross War Memorial Children's Hospital (RXH)

Lizelle Clark (Department of Radiology,University of Cape Town), Tracy Kilborn, Nicky Wieselthaler (RXH)

Paediatric cardiac imaging is a complex, challenging and highly specialised field that has been revolutionised by newer imaging techniques in the form of multidetector computed tomography angiography(MDCTA)and magnetic resonance (MR) cardiac imaging. These modalities have emerged to play dominant roles in the assessment of congenital heart disease, challenging conventional forms of imaging. It is clear from the literature that the roles of these newer imaging modalities have been established in developed countries; however, their roles in the developing world remain limited owing to lack of infrastructure and resources. At present, our institution does not offer a cardiac MR imaging service. Echocardiography remains the primary modality for the diagnosis of cardiac disease, predicting the need for surgery in the majority of cases. Cardiac catheterisation and cardiac CTA are reserved for a subset of patients. In this retrospective study, we evaluate our initial experiences using a 64-slice Phillips MDCT to answer specific questions raised by inconclusive echocardiography and cardiac catheterisation findings. From December 2009 to April 2011, a total of 35 children underwent cardiac CTAs. All patients had echocardiography imaging. Fifty-four per cent of patients underwent conventional cardiac angiography. In our case series, CTA proved to be effective in the evaluation of structural abnormalities not adequately defined - particularly with regard to the pulmonary arteries, major aorto-pulmonary collateral arteries, extra-cardiac anatomy and patency of shunts. Cardiac CTA in our setting is feasible, accurate, reproducible and objective, and we recommend synergistic use of echocardiography and CTA in selected cardiac patients.

Free fluid on 64-MDCT after blunt abdominal injury in a trauma referral centre in KwaZulu-Natal: A profile of incidence and outcome

Damon Surjit Jeetoo, Frederik Buitendag (Department of Radiology, Pietermaritzburg Metropolitan Hospitals Complex), Damian Clarke, Thomas Walker (Department of Surgery, Pietermaritzburg Metropolitan Hospitals Complex

Introduction and background. Free fluid seen in the absence of solid organ or hollow viscus injury poses a diagnostic dilemma for both radiologists and trauma surgeons. We looked at the incidence of isolated free fluid in the context of a busy South African trauma referral centre (Edendale Hospital) in KwaZulu-Natal. We identified and followed retrospectively and prospectively 121 patients with blunt abdominal injury who had a CT of the abdomen and pelvis upon first presentation, within a period of 20 months from September 2009 to April 2011.

Results. There were 36 cases of free fluid in the abdomen and pelvis on CT. Of these, 21 included solid organ injuries. There were 7 cases with hollow viscus injuries and 8 cases with isolated free fluid. Of the latter group, 3 had negative laparotomies, 4 were managed conservatively, and 1 was lost to follow-up.

Conclusion.The studied patients who sustained blunt abdominal trauma and were diagnosed with isolated free fluid in the abdomen or pelvis on a 64-MDCT did not require laparotomy and could have been managed conservatively.

Multi-detector computed tomography venography (MDCTV) as a diagnostic tool in the management of patients with atypical, complicated and/or recurrent varicose veins

Andrew Lawson (University of Cape Town, Groote Schuur Hospital), Paul Rischbieter (general practice), Tushar Peedikayil, Jeannine Owen, Steve Beningfield (Groote Schuur Hospital)

Purpose. To evaluate the role of MDCTV as a diagnostic tool in the management of patients with atypical, complicated and/or recurrent varicose veins when compared with conventional venography.
Materials and methods. A retrospective review of 21 patients who had undergone both MDCTV and conventional transfemoral or transpopliteal venography between January 2008 and April 2011 for the management of recurrent varicose veins and/or chronic venous ulcers. MDCTV was performed using a Siemens Emotion Somatom 16-slice CT scanner. Spiral acquisition was commenced 180 seconds after intravenous injection of 150 $\mathrm{ml}$ of $350 \mathrm{mmol} / \mathrm{l}$ iodinated contrast medium. A reconstruction interval of $1.5 \mathrm{~mm}$ was used. Conventional venography was performed by the resident vascular surgeon and was followed by stenting or coiling where appropriate.

Results. MDCTV and venography were compared in 21 patients (6 males, 15 females; average age 55 years, range $33-78$ years); 8 also underwent endovascular iliac vein stenting. The area under the receiver operator curve (ROC) curve for percentage iliac vein stenosis determined on MDCTV v. venography was 0.75 . Four false-positive (19\%) iliac vein stenoses were reported on MDCTV. Ten patients underwent gonadal vein coil embolisation. Gonadal vein size $>5.2 \mathrm{~mm}$ (range $1-11 \mathrm{~mm}$ ) on MDCTV predicted significant venographic reflux requiring coil embolisation. Three (30\%) patients who underwent embolisation did not have gonadal vein enlargement on MDCTV.

Conclusion. MDCTV plays an important adjunctive role in the diagnostic workup of patients with complex venous disease. The findings at MDCTV correlate well with conventional venography.

The prevalence of missed pathology on normal non-contrast-enhanced cranial computed tomography

Cornelia Minne (University of Limpopo), Farhana E Suleman, Nonjabulo Z Makhanya, Margaret E Kisansa, Nazema Ebrahim (University of Pretoria)

Objective. To determine the prevalence of missed pathology on normal noncontrast-enhanced cranial computed tomography.

Method. Cranial computed tomography scans over a 12 -month period were retrospectively reviewed by 3 readers. The non-enhanced CT (NECT) and contrast-enhanced CT (CECT) scans were read at separate occasions and readers blinded to the history, each other's interpretation and their own interpretation of the NECT. Interpretation discrepancies between readers were resolved during a meeting where consensus was reached. Cases with missed pathology on the NECT were also evaluated retrospectively at a joint meeting between the 3 readers to determine whether the pathology was visible on the NECT.

Results. In this study, 3.28\% of cases had pathology missed by 3 readers on the NECT. Re-evaluation by the panel reduced this to $1.42 \%$, indicating a reading error of $1.85 \%$.

Conclusion. Patients with a normal NECT, no neurological clinical signs, no history of tuberculosis, and no known tumours or risk factors for venous thrombosis have a very small chance of missed pathology when using only NECT. Omitting unnecessary CECT will in turn reduce the risk of intravenous iodinated contrast and radiation exposure to the patient and running cost, as well as increase the throughput of patients. The alternative of doing only NECT will reduce radiation exposure and the risk of missing pathology.

How we eyeball the small bowel: Newly introduced CT enteroclysis at Tygerberg Hospital

Braham van der Merwe (University of Stellenbosch and Department of Radiology, Tygerberg Hospital), B van der Merwe, H Els, A Maydell, S Scheepers (Division of Radiodiagnosis, Tygerberg Hospital))

The imaging and diagnosis of small bowel disease is a complex issue. The previous gold standard of fluoroscopy utilising barium as positive contras medium has numerous limitations and is increasingly being replaced with cross-sectional imaging. Both computed tomography enteroclysis (CTE) and magnetic resonance enterography (MRE) offer the advantage of imaging the bowel lumen as well as extraluminal tissues. The latter is not achievable with conventional fluoroscopic examinations and has important management 
implications, especially for conditions such as Crohn's disease. CTE is relatively available, but the technique described in the literature proposes utilising methyl cellulose via infusion pumps as enteral negative contrast. This enteral agent is not available in South Africa and the technique is quite costly, which is a further hindrance in our resource-constrained setting. We have solved these problems with a creative and unique technique not previously described. It is an inexpensive solution using equipment already at our disposal and available in most hospital settings. The bowel distension is adequate for diagnostic purposes, and we believe the image quality to be on a par with that published in the literature. We present a number of diverse cases demonstrating various small bowel pathologies to this end.

Findings on CT brain scans of patients referred with an index psychotic episode

Matthys Van Wyk (University of the Witwatersrand)

Introduction. Psychosis is the inability to separate subjectivity and reality and can be functional or organic. Organic psychoses are attributed to physical brain conditions, excluded by imaging. CT scanning requires evaluation regarding the detection of organic pathology in South Africa, with its high TB and HIV prevalence and resource limitations.

Aim. To determine the prevalence of structural brain abnormalities detected on CT in patients referred with a first-episode psychosis in a South African population.

Materials and methods. CT scans of the brain in patients referred after a first-episode psychosis (as per protocol, at one institution) were reviewed retrospectively over a 2-year period. Demographic data, HIV status, spaceoccupying lesions (SOL), ventricle size, abnormal calcifications and features of raised intra-cranial pressure were recorded.

Results. Of 112 scans reviewed (51.79\% males; mean age 37 yrs; range 16 - $67 \mathrm{yrs}), 11.61 \%$ tested HIV-positive. A total of 21 (18.75\%) patients had abnormalities on CT, $2(1.78 \%)$ of which were significant requiring further investigations. No SOLs were detected. Only $4(3.57 \%)$ patients had enlarged but not hydrocephalic ventricles. Nine $(8.03 \%)$ patients had ageinappropriate involutional changes. Seven $(6.25 \%)$ patients had old calcified granulomata. No scans showed features of raised intra-cranial pressure.

Conclusion. The yield of abnormalities in CT brain scans in patients referred with a first-episode psychosis at our institution is high. However, only a small percentage of these are significant, and can be attributed to the psychosis or affect management.

\section{Poster presentations}

(alphabetical order according to presenting author (bold font))

Paraduodenal hernias exposed

Ernst Boshoff, Matthew Goodier (University of the Witwatersrand)

Baseline chest radiograph appearances of HIV-infected children eligible for anti-retroviral therapy

Vicci du Plessis, Aisne Stoker (Grey's Hospital), Savvas Andronikou (University of the Witwatersrand), Gabriel Struck (Stony Brook University School of Medicine), Neil McKerrow (Pietermaritzburg Metropolitan Hospitals)

\section{An atypical appearance of pulmonary metastases}

Jacqueline du Toit (Stellenbosch University Radiology Department, Tygerberg Hospital), Pieter Barnardt (Clinical Oncology, Tygerberg Hospital)

Adequacy of paediatric renal tract ultrasound

Nishentha Govender (University of the Witwatersrand)

Severe coarctation of the aorta v. interrupted aortic arch: A case of severe aortic obstruction in a 24-year-old woman with refractory hypertension Damon Jeetoo, Vicci Du Plessis (Department of Radiology, Pietermaritzburg Metropolitan Hospitals Complex)
The many faces of lymphobronchial TB

Susan Lucas, Savvas Andronikou (University of the Witwatersrand), Pierre Goussard, Robert Gie (University Stellenbosch)

The many faces of hydatid in South Africa

Nasreen Mahomed, Matthew Goodier, Michelle Wong, Grace Rubin (University of the Witwatersrand)

Disseminated rhabdomyosarcoma with spinal metastases

Nasreen Mahomed, Sugeshnee Pather (University of the Witwatersrand)

Unusual suprasellar presentation of an atypical teratoid/rhabdoid tumour in a child

Nasreen Mahomed, S Dlangamandla, S Pather (University of the Witwatersrand)

Non-accidental injury - a South African experience

Nasreen Mahomed, Matthew Goodier (University of the Witwatersrand)

Brain computed tomography perfusion imaging for acute stroke: University of the Free State's experience

Frans Naude, Werner Harmse (University of the Free State)

The role of multidetector computed tomographic angiography (MDCTA) in pre-endovascular interventional decision making

Tushar Peedikayil, Andrew Lawson, Stephen Beningfield (Groote Schuur Hospital/UCT)

Supracardiac total anomalous pulmonary venous connection Tanyia Pillay, Firoza Motara (Rahima Moosa Hospital)

Can you diagnose the different congenital cardiac anomalies? Tanyia Pillay (Rahima Moosa Hospital)

Name this vessel - A rare primitive carotid-basilar anastomosis Amaresh Ranchod (Department of Radiology, University of the Witwatersrand, Chris Hani Baragwanath Hospital), Ronel Swartz, Savvas Andronikou, Victor Mngomezulu (Department of Radiology, University of the Witwatersrand), Shaheed Gora (Department of Neurology, University of the Witwatersrand)

A case of complete androgen insensitivity syndrome - clinical and imaging findings

Shirley Sadler, Ronald James Urry, Fazleh Mahomed (Grey's Hospital, Pietermaritzburg)

Hydatid disease: here, there and everywhere

Shaun Scheepers, Hein Els, Arthur Maydell, Braham van der Merwe (Stellenbosch University)

Impending aortic rupture: Four signs to keep you out of trouble Shaun Scheepers, Hein Els, Arthur Maydell, Braham van der Merwe (Stellenbosch University)

Vanishing white matter disease, MRI imaging over 4 years Werner Steyn (University of the Witwatersrand)

Ovarian vein thrombosis: common CT findings of an uncommon entity Braham van der Merwe (Stellenbosch University, Tygerberg Radiology)

Radiology of the cutting edge

Braham van der Merwe (Stellenbosch University, Tygerberg Radiology) 